\title{
Pigeons' discrimination of color proportion in computer-generated visual displays
}

\author{
JACKY EMMERTON \\ Purdue University, West Lafayette, Indiana
}

\begin{abstract}
Pigeons were trained to discriminate the proportion of red to green color in paired stimulus displays. Initially, the stimuli were horizontal bars composed of continuous blocks of color that varied from being all red versus all green to .5 proportions of these two colors. Discrimination accuracy decreased as a function of the disparity in the proportions of the two colors. This relationship was maintained when the stimulus configurations were altered in various ways. Tests with horizontal bars indicated that the pigeons could utilize differences in the lengths (or areas) of one of the colors when choosing between stimuli. They did not rely only on this type of cue to assess proportion disparities but rather on multiple stimulus parameters. Also, the form of the discrimination function suggests that the pigeons distinguished ratio differences, so that Weber's law applies to this type of discrimination.
\end{abstract}

One question in animal cognition is how organisms differentiate the more abstract properties of the environment, especially space, time, and number (Gallistel, 1989; Pearce, 1997; Roberts, 1998). The quantity of a useful resource, such as food, could be derived from any combination of these properties (e.g., the spatial extent of a food patch at any one time, the number of items available simultaneously, or the amount that can be obtained within a fixed time). Information about these dimensions is probably as fundamental as sensory data about differences in sound frequency or light intensity. In order to show that an animal can utilize changes in such abstract dimensions, the stimuli in tests of discrimination must be carefully controlled for potentially confounded characteristics. If, for instance, an animal really responds to the number of items it sees or hears, it must maintain its behavior when the size or shape of visual items is altered or when the duration of a series of sounds is manipulated. A variety of studies on numerical and temporal discriminations have incorporated such controls (e.g., Brannon \& Terrace, 2000; Emmerton, Lohmann, \& Niemann, 1997; Honig, 1992; Meck \& Church, 1983; Roberts \& Mitchell, 1994).

The present study examined how pigeons discriminate relative quantity. Specifically, visual displays were generated in which the proportion of different colors comprising these stimuli was varied. In previous experiments with pigeons, Honig and his colleagues (Honig, 1992; Honig \& Stewart, 1989, 1993) equated proportion with the relative numerosity of different elements that comprised successively presented visual arrays. They showed that the discrimination decreased as the differences in the

Some of these data were presented at the 37th Annual Meeting of the Psychonomic Society in Chicago, November 1996. Correspondence should be addressed to J. Emmerton, Department of Psychological Sciences, Purdue University, West Lafayette, IN 47907-1364 (e-mail: jemmert@psych.purdue.edu). relative numbers of elements diminished. The main purpose of the present study was to investigate whether pigeons discriminate proportion under a variety of stimulus conditions, most of which differed from the numerosity arrays used by Honig. The starting point was to present the pigeons with paired stimuli in which the relative quantities of continuous blocks of color were manipulated. The initial question was whether, like Honig's animals, their accuracy in discriminating depended on changes in the proportion of the colors in the stimuli. A further aim was to see whether pigeons would maintain a discrimination of proportion when the form of the stimuli was changed substantially to include both arrays similar to the type used by Honig and other configurations that have not been used before. Another aim was to examine whether the pigeons' choices were based on differences in specific parameters of the stimuli, such as the lengths or areas of component parts.

Finally, the characteristics of the discrimination function itself were considered. Other research suggests that Weber's law applies not just to discriminations of sensory intensities but also to animals' responses to temporal or numerical differences (Fetterman, 1993; Gibbon, 1977; Meck \& Church, 1983). Discrimination accuracy is then a function of the relative differences, expressed as ratios, rather than the absolute differences in the temporal duration of events or their numerosity. The expectation in the present study was that choices between other aspects of quantity would similarly conform to Weber's law. This could indicate a commonality in the way that a variety of stimulus properties are processed.

\section{EXPERIMENT 1}

Experiment 1 was designed to establish the pigeons' discrimination of complementary proportions of red and green colors in paired horizontal bars and then to investi- 
gate the effects on discrimination of various stimulus manipulations.

The first manipulation was to change bar orientation. Although pigeons show rotational invariance and recognize geometric figures when stimulus orientation is altered (Hollard \& Delius, 1982), it is not clear whether orientation changes would affect discrimination accuracy in the present task. Humans, for instance, show a horizontal/ vertical illusion in which judgments of length are affected. However, this illusion - in which a vertical length is seen as longer than a physically equal horizontal length-is best perceived when a vertical line and a horizontal line form an inverted T (Frisby, 1979). Next, the horizontal bars were broken up into small blocks of color and were configured so that the lengths of colored portions could not easily be discriminated. Finally, the stimuli consisted of arrays of small rectangles, arranged either as regular matrices or in a slightly irregular way to break up potential texture effects. The regular arrays resembled the types of stimuli used by Honig and his colleagues to investigate numerosity discrimination (Honig, 1992; Honig \& Stewart, 1989, 1993). The aim of this stimulus condition was twofold. First, it was designed to test whether the pigeons transferred their discrimination performance to stimuli configured in this novel way. Second, the purpose was to see whether, like Honig's birds, these pigeons discriminated color proportion when this parameter was equivalent to the relative numerosity of two types of elements comprising the arrays.

\section{Method}

\section{Subjects}

Six adult pigeons (Columba livia) of mixed breed served as subjects. They had prior experience with operant conditioning procedures, but the stimuli, presented behind pecking keys, had been simple animation displays composed of white dots. The pigeons were housed in individual cages in a colony room with a 14:10-h light:dark cycle. They were maintained at $80 \%-85 \%$ of their ad-lib body weights. Water and grit were freely available in their home cages.

\section{Apparatus}

The operant conditioning chamber, of in-house design, measured $33 \times 34.5 \times 32 \mathrm{~cm}$. One side gave access, through a $26.5 \times 15 \mathrm{~cm}$ open area, to an adjoining Zenith 1492 FTM (flat-screen) VGA monitor equipped with a Carroll Touch scanning infrared touch screen. A 1-mm-thick sheet of clear plastic protected the screen and cushioned the impact of the pigeons' pecks. On each side wall was an opening $(3.5 \times 5.5 \mathrm{~cm})$ to a Colbourn Instrument (Model E1410) grain feeder. The two feeders could be illuminated separately and independently of food delivery. An infrared light gate, added to each unit, sensed when a pigeon put its head into the opening. A $\mathrm{dim}, 1.2-\mathrm{W}$ houselight was positioned to the rear of the box. It was shielded to prevent light reflections interfering with the monitor displays. A PC computer generated the visual displays, recorded responses via the touch screen, and controlled events in the chamber via an interface. Visual stimuli were generated using the graphics library of Borland TurboPascal. During the main part of the experiment, a pair of stimuli was presented on each trial. The centers of the left and right stimulus displays remained the same across stimulus conditions and were aligned $11.3 \mathrm{~cm}$ apart and $14.2 \mathrm{~cm}$ above the chamber floor. Details of the stimuli are given below.

\section{Procedure}

Response training. The pigeons were trained for two sessions with a modified autoshaping procedure (Brown \& Jenkins, 1968) to peck a white cross-hatched circle in the middle of the monitor screen. The circle was 30 pixels in diameter (equivalent to $11.5 \mathrm{~mm}$ on the screen). A peck was sensed by the touch screen if it fell within a target area of approximately $24 \times 26 \mathrm{~mm}$, centered on the stimulus. A hopper light was switched on, signaling the availability of food, at the end of a 6-sec stimulus period, unless the pigeon pecked the stimulus within this time. In that case, the stimulus was erased, and the hopper light came on immediately. As soon as the pigeon inserted its head in the feeder opening, the hopper was activated for $3 \mathrm{sec}$, then the feeder and hopper light were switched off. If the pigeon did not put its head in the opening, the light went out after $20 \mathrm{sec}$. Over the 48 trials of a session, reinforcement was randomly available from the left or right hopper on each trial. There was a 15 -sec intertrial interval (ITI) between trials.

Discrimination training. Throughout this study, a simultaneous discrimination procedure was used. On each trial, a pair of stimuli was displayed on the monitor. A single peck to one of them extinguished the display. If the correct stimulus $(\mathrm{S}+)$ was pecked, the hopper light adjacent to the $\mathrm{S}+$ side was switched on. When the pigeon inserted its head in the feeder opening, the hopper was activated for $2 \mathrm{sec}$. If the pigeon failed to do this, the feeder was darkened after $20 \mathrm{sec}$. If the pigeon pecked at the incorrect stimulus ( $\mathrm{S}-$ ), a 5 -sec time-out ensued with the houselight off. Following reward or timeout, there was a 5-sec ITI with the houselight on.

First, the pigeons were trained to discriminate a red horizontal bar from a green horizontal bar, presented on a dark background. Each bar was 50 pixels long and 10 pixels wide (equivalent to $19 \times$ $3.8 \mathrm{~mm}$ ). For 3 pigeons, red was the $\mathrm{S}+$ and green was the $\mathrm{S}-$. For the other 3 pigeons, these contingencies were reversed. A trial was terminated by a single peck within a $30 \times 39 \mathrm{~mm}$ target area encompassing each stimulus. Food reward or time-out followed. If a trial ended in time-out, the trial was repeated until a correct response was made. Data from these correction trials were recorded but were not used in computing the discrimination scores. There were 48 noncorrection trials per session plus any correction trials. The side on which the $\mathrm{S}+$ was presented varied quasi-randomly across trials (Fellows, 1967). Sessions were repeated until the pigeon scored $\geq 90 \%$ correct choices on two consecutive sessions.

Varied proportions of red:green in horizontal bar stimuli. For five sessions, the proportions of red:green within the paired bars varied across trials. Each session began with 10 "warm-up" trials with bar stimuli in uniform colors. An incorrect choice initiated a correction procedure. In the other 96 trials per session, correct and incorrect choices led to reward and time-out, respectively, but there was no correction procedure. The $\mathrm{S}+$ was the stimulus of a pair with the greater proportion of the positive color (i.e., the color that was correct for a bird in discrimination training). For S+ stimuli, this proportion varied between 1.0 and .5 in steps of .1. The corresponding $\mathrm{S}-$ stimuli contained the complementary proportion of positive color. On trials in which both stimuli had .5 proportion values, one stimulus was arbitrarily defined as the $\mathrm{S}+$. These trials provided a built-in control to check whether the pigeons performed at chance when the color proportions were identical. There were 16 trials at each of the six proportion values. The same proportion values and reinforcement contingencies were used throughout Experiment 1 .

In addition to variations in the proportion of red:green, the positions of the color components at the left or right end of S+ and Svaried in four configurations: $\mathrm{L} / \mathrm{L}, \mathrm{R} / \mathrm{R}, \mathrm{L} / \mathrm{R}$, and $\mathrm{R} / \mathrm{L}$ (with reference to the positive color's position). For each proportion value, the $\mathrm{S}+$ was presented equally often on the left and right sides. The order of stimuli combining these three attributes (color proportion, configuration, and $\mathrm{S}+/ \mathrm{S}-$ location) was quasi-random and was controlled 
by two different sequences that were alternated across sessions. Figure 1 shows examples of stimuli with these attributes.

The position of the pigeon's peck was registered as a pair of $x$ and $y$ coordinates on the touch-screen matrix. The touch screen was operated in enhanced mode so that a shift in averaged peck location could be registered with an accuracy of $3 \mathrm{~mm}$.

Changes in stimulus orientation. Each stimulus bar was $50 \times$ 10 pixels in size with a $30 \times 32 \mathrm{~mm}$ touch-sensitive target area. Across trials, the stimulus attributes of color proportion (six values), bar orientations (four combinations), stimulus conf igurations (four codes), and left/right location of the S+ (two codes) varied. In order to counterbalance all these combinations, 192 trials were needed. To avoid satiation, full counterbalancing was achieved across pairs of sessions, and the data from each pair were combined. Each session thus consisted of 10 warm-up trials, followed by 96 trials with varying stimulus combinations. Both bars were horizontally oriented on half of the warm-up trials and were vertically oriented on the other half. Color proportions varied in the remaining trials. The orientation combinations of horizontal and vertical were $\mathrm{H} / \mathrm{H}, \mathrm{V} / \mathrm{V}, \mathrm{H} / \mathrm{V}$, and $\mathrm{V} / \mathrm{H}$ for the $\mathrm{S}+\mathrm{S}-$. Stimulus configuration also varied so that the positive color appeared at the left or right end of a horizontal bar and at the top or bottom of a vertical bar. The order of presentation of stimuli with the various attribute combinations was controlled by four different quasi-random sequences that were changed daily. Eight sessions (combined to give four pairs for data analysis) were completed.

Horizontal bars composed of discrete blocks of colors. The stimuli were horizontal bars, but now each bar was composed of 20 small blocks of color, each 10 pixels long and 2 pixels wide. There was a gap of 1 pixel, filled by the black background color, between adjacent blocks. The use of video-paging set the monitor to EGA mode. Thus, each bar was $22.5 \mathrm{~mm}$ in overall length $\times 5.2 \mathrm{~mm}$ wide, within a $36 \times 39 \mathrm{~mm}$ target area.

There were six sessions, with 102 trials per session. The first 6 trials were warm-up trials in which one bar was red and the other green, but both bars consisted of small blocks. In the remaining 96 trials, half had the same-color blocks in each bar arranged consecutively. In mixed-color stimuli, there were four configurations of the red or green series of blocks (L/L, R/R, L/R, and R/L). For the other 48 trials, the color blocks were arranged randomly. For each block arrangement, there were six proportion values with 8 trials per value and two $\mathrm{S}+$ positions per trial. Some examples of stimuli with the two types of block arrangement are shown in Figure 2. The different stimulus combinations were presented in quasi-random order according to two different sequences, each of which was used on three randomly chosen sessions.

Arrays of colored rectangles. Each stimulus was an array of 30 small rectangles, each $5 \times 5$ pixels in size. With video-paging, each element and the overall matrix were slightly longer on the $y$ axis than on the $x$ axis. For the regular arrays, the rectangles were arranged in a $6 \times 5$ matrix that measured $21.4 \times 23.2 \mathrm{~mm}$ on the screen. For the irregular arrays, the coordinates of the 30 rectangles were constant, but their arrangement did not follow a regular matrix. The overall dimensions of these arrays were about $24.2 \times$ $30.4 \mathrm{~mm}$. The target area for each array was $36 \times 42 \mathrm{~mm}$. Allocation of a red rectangle or a green rectangle to each of 30 possible locations was random. The proportion of red:green was varied by altering the numbers of each type of element within an array. Examples of both types of stimulus are shown in Figure 3

There were six sessions, with 104 trials per session. The first 8 were warm-up trials with arrays of uniform color. Arrays were regular on 4 trials and irregular on the others. On 48 of the remaining trials, the stimulus arrays were regular, on the other 48 trials, they were irregular. The proportion of red:green rectangles varied over six values. $\mathrm{S}+$ position was counterbalanced across trials. Trials with these parameter combinations were ordered quasi-randomly according to two different sequences that were alternated daily.

\section{Results}

All the pigeons learned to peck reliably and to take food promptly in the response training sessions. They learned the discrimination of red versus green bars quickly and performed above chance during the first session of discrimination training. They achieved the training criterion within two to four sessions ( $M=2.7$ sessions). In all subsequent stimulus conditions, average performance on warm-up trials always exceeded $94 \%$ correct. Data analyses refer to performance on the remaining trials.

\section{Varied Proportion of Red:Green in Horizontal Bar Stimuli}

Data were analyzed with repeated measures analyses of variance (ANOVAs). In these and other statistical analyses, an alpha level of .05 was used. Since the configuration of color components was not applicable to the pure color bars, a two-way (bar configuration $\times$ sessions) ANOVA was performed first on data from the 80 trials per session in which stimuli had mixed color components. Bar configuration had no significant effect on performance level $(F<1)$. There was a slight but reliable improvement in discrimination performance across sessions $[F(4,20)=4.04]$. Data from all 96 trials were then analyzed with proportion value as one factor (with scores for different configurations collapsed over each proportion level) and sessions as the other factor. Stimulus proportion had a highly significant effect on discrimination accuracy $[F(5,25)=50.54]$. Discrimination scores again increased slightly across sessions $[F(4,20)=3.34]$. Due to some improvement across sessions in discrimination of just the intermediate values of stimulus proportion, the interaction between proportion value and sessions was also significant $[F(20,100)=2.42]$.

The function relating percent correct discrimination to stimulus proportion was nonlinear, so a logit transformation was applied to the mean discrimination data to linearize them. The logit scale is defined as $\ln [\%$ correct/ $(100-\%$ correct)] (Macmillan \& Creelman, 1991). The stimulus values were converted to disparity ratios, computed as the differences in proportion of positive color in the $\mathrm{S}+$ and $\mathrm{S}-$ stimuli divided by the sums of those proportion values. Since the proportions of positive color in the $\mathrm{S}+$ and $\mathrm{S}-$ stimuli were complementary in this experiment, the denominator in this calculation of disparity ratio was equal to 1 . A linear regression analysis performed on the transformed data yielded a highly significant coefficient of determination $\left(r^{2}=.99\right.$; Figure 1). Similar transformations were performed on the mean data for individual pigeons. Coefficients of determination for the individual regression functions varied between .85 and .98 and were significant in each case.

\section{Changes in Stimulus Orientation}

Since the configuration factor had no effect on discrimination accuracy in the previous condition, data were pooled across configurations. Then, a three-way (proportion $\times$ orientation $\times$ pairs of counterbalanced sessions) ANOVA was performed. Color proportion had 


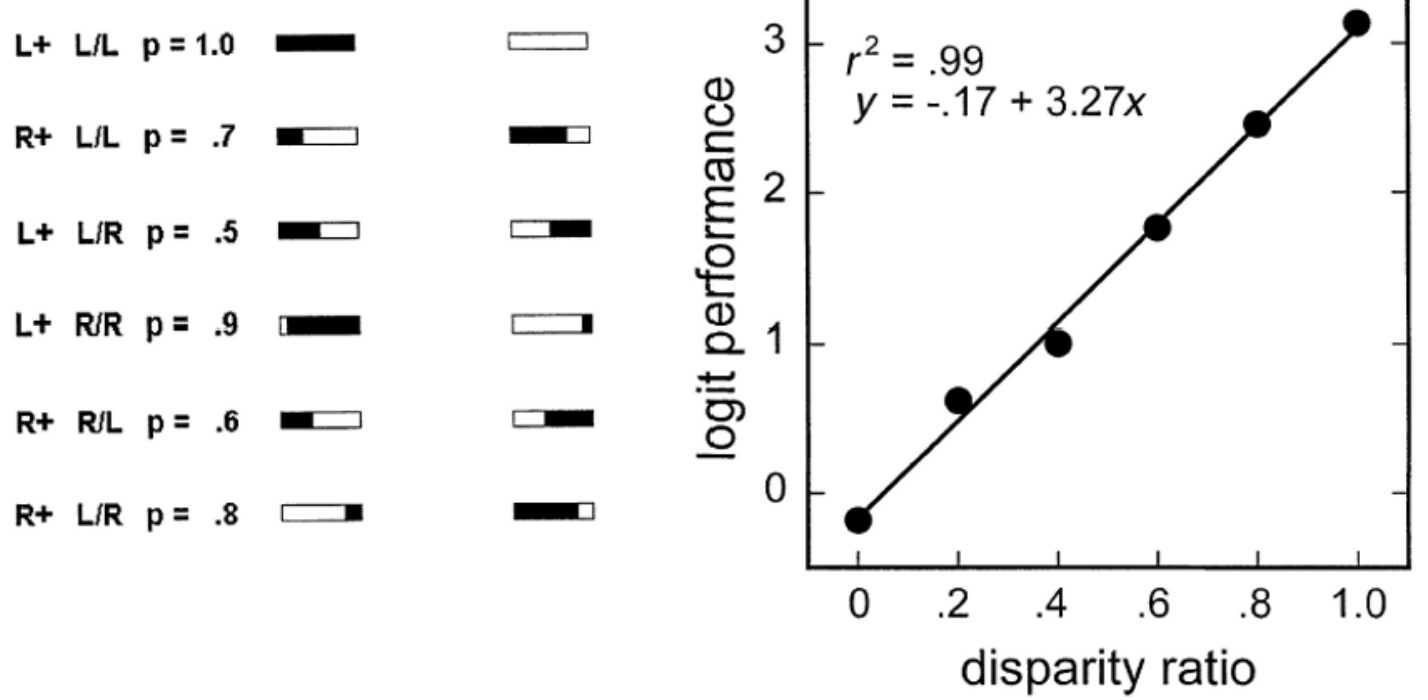

Figure 1. Left side: Examples of the solid, horizontal bar stimuli used in the first stimulus condition of Experiment 1 . The black portion of a stimulus represents the positive color, and the white portion represents the negative color. Abbreviations indicate stimulus attributes. $L+$ and $R+$ indicate left and right positions of $S+; L / L, R / R, L / R$, and $R / L$ indicate positions within $S+/ S-$ of the positive color; $p$ indicates the proportion of positive color in $S+$. Right side: Linear regression of discrimination performance, transformed to a logit scale, versus the disparity ratio of positive-color proportions in paired stimuli.

a highly significant effect on discrimination accuracy $[F(5,25)=67.29]$. Bar orientation had a smaller but significant effect on performance level $[F(3,15)=5.16]$. Newman-Keuls post hoc tests revealed that this was mainly due to differences in discrimination accuracy on trials in which the stimulus bars were arranged orthogonally. Discrimination was best when the S+ was horizontal and the $S-$ was vertical, and it was worst for the opposite combination. There were no reliable changes in performance across paired sessions $(F<3)$. The only interaction that was significant was for proportion $X$ orientation $[F(15,75)=2.11]$. This result was mainly due to the pigeons' choices at the lowest proportion values when they discriminated orthogonal orientations. When color proportions were identical (proportion value $=.5$ ), responding was slightly biased toward choosing the horizontal bar in the $\mathrm{H} / \mathrm{V}$ pair. There was a similar bias when the orientation combination was $\mathrm{V} / \mathrm{H}$, thus leading to more incorrect guesses with these otherwise equivalent stimuli. When the stimuli were difficult to discriminate on the basis of proportion (proportion of positive color in $\mathrm{S}+=$ .6), choice accuracy was slightly better when the $S+$ was horizontal and the $S-$ was vertical, compared with the other three orientation combinations.

There was a linear relationship between logit performance and disparity ratios of proportion $(y=.09+4.45 x)$, with a significant coefficient of determination $\left(r^{2}=.96\right)$.

\section{Horizontal Bars Composed of Discrete Blocks of Colors}

The ANOVA assessed the effects of block arrangement $X$ proportion $X$ sessions. Only color proportion had a significant effect on choice accuracy $[F(5,25)=87.59]$. Linear regression analyses on the logit-transformed performance scores and proportion disparity ratios yielded significant coefficients of determination $\left(r^{2}=.99\right.$ for the consecutive arrangement, and $r^{2}=.94$ for the random arrangement of blocks; Figure 2).

\section{Arrays of Colored Rectangles}

In a three-way (array configuration $\times$ proportion $\times$ sessions) ANOVA, only the variation in proportion had a significant main effect on discrimination accuracy $[F(5,25)=82.03]$. The only other term that was significant was the three-way interaction $[F(25,125)=1.83]$. The relationship between transformed discrimination scores and proportion disparities was significant with coefficients of determination $\left(r^{2}=.95\right.$ for regular arrays, and $r^{2}=.98$ for irregular arrays; Figure 3 ).

\section{Discussion}

Discrimination performance in all stimulus conditions was highly dependent on variations in the proportion of colors contained in the paired stimuli. This relationship was particularly apparent when the discrimination scores were transformed to a logit scale and the differences in stimulus values were expressed as disparity ratios. A scale of disparity ratios, rather than simple proportion differences, was used since it was expected that the discrimination functions would conform to Weber's law. But, with complementary proportion values in $\mathrm{S}+$ and $\mathrm{S}-$ stimuli, either proportion scale could have been used. The appropriateness of the disparity ratio scale will become clearer in Experiment 2. 


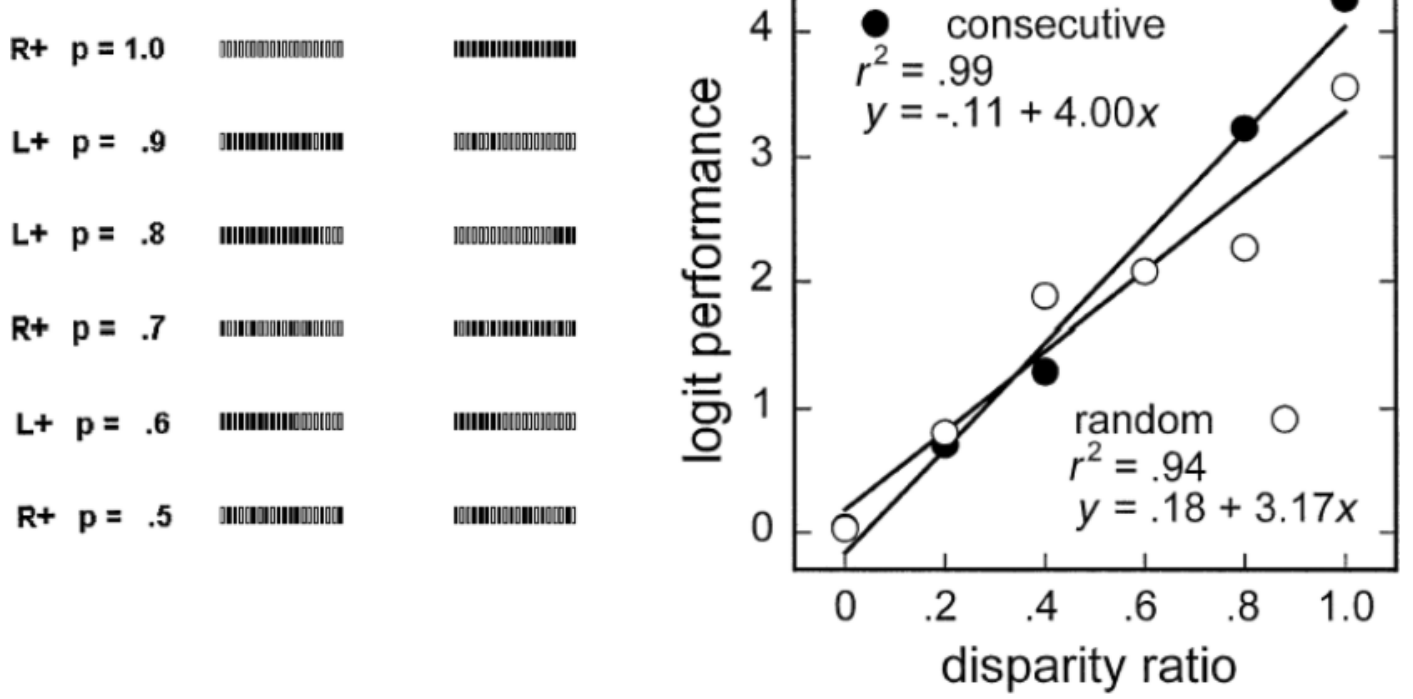

\begin{abstract}
Figure 2. Left side: Examples of discrete-block stimuli. Alternating rows illustrate the two arrangements, consecutive and random, of same-color blocks within stimulus pairs. $R+$ and $L+$ indicate right or left positions of the $S+$ stimulus; $p$ gives the proportion of positive color within the $S+$. Right side: Linear regression functions of logit discrimination performance versus disparity ratio of proportions for the consecutive and random arrangements of positive- and negative-colored blocks.
\end{abstract}

Altering the orientation of the solid-bar stimuli had an effect on discrimination accuracy that, although not striking, proved to be statistically reliable. The best mean performance was obtained for one combination of orthogonal orientations ( $\mathrm{S}+$ horizontal and $\mathrm{S}-$ vertical), and the poorest mean score was obtained for the opposite orientations ( $\mathrm{S}+$ vertical and $\mathrm{S}-$ horizontal). However, this was mainly due to a slight bias toward choosing the horizontal bar when discrimination of proportion became difficult (proportion value $=.6$ ) or impossible (proportion value $=.5$ ). This suggests that stimulus orientation per se had very little effect on proportion discrimination. The bias is the opposite of what would be expected if the pigeons had experienced a vertical/horizontal illusion. The source of the response bias remains speculative. The pigeons may simply have defaulted to choosing the more familiar stimulus orientation when discrimination of proportion became difficult. Another idea was considered but not corroborated. This was that the pigeons might try to "grasp" the bar across its width, as they would when picking up a feather (own observations), and might find this maneuver more difficult with vertical stimuli. However, direct observations of the pigeons' behavior in the training chamber indicated that it was highly variable whether or not they tilted their heads to peck. Head tilting was no more likely with vertical stimuli than with horizontal stimuli.

Solid-bar stimuli were constant in length, and the proportions of positive and negative colors were complementary within stimulus pairs. So the pigeons could have based their discrimination on differences in length or else area of the positive-color portions alone, rather than discriminating the relative proportions of mixed colors. The latter possibility was addressed in subsequent experiments. But results with stimuli consisting of discrete elements suggested that discrimination was not based just on the lengths of continuous blocks of color.

First, the pigeons were presented with bars composed of discrete blocks of color. Altering the overall lengths (as well as the widths) of these bars and the way their component colors were arranged had no noticeable effect on the pigeons' ability to discriminate changes in the color proportions. Although the pigeons might still have discriminated the relative lengths of consecutively ordered blocks of the same color, they could not have done so with bars consisting of randomly arranged blocks. However, these stimuli did not preclude discrimination of the overall areas of red and green in each stimulus. The pigeons could have integrated the total areas across separate blocks of red and green, even when these elements were randomly intermixed. If they did integrate colored areas, they apparently did not have to learn to do so, since discrimination performance did not improve across sessions.

Another change in stimulus configuration was the use of arrays of small rectangles. Although this stimulus condition precluded discrimination of lengths, the rectangles were always of the same fixed size. So, the pigeons could still have integrated the areas of randomly distributed red or green elements in each array and then based their choices on a comparison of the areas of one or other color.

\section{EXPERIMENT 2}

In Experiment 1, the proportions of positive and negative colors were always complementary in each S+ and $\mathrm{S}-$ pair; however, in analyzing the relationship between 


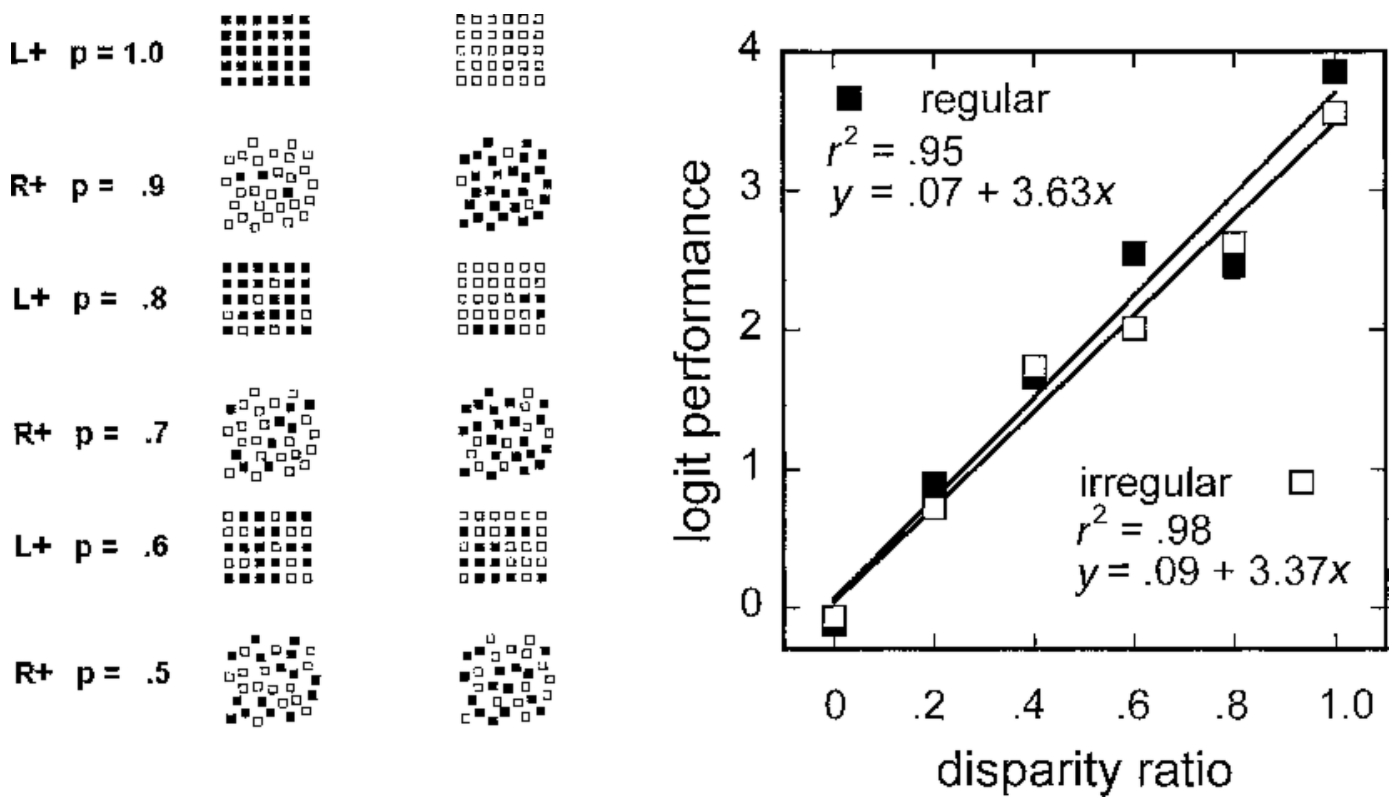

Figure 3. Left side: Examples of stimulus arrays. Alternating rows show regular and irregular arrays. The arrays, as well as their elements, were slightly more rectangular on the screen than they appear in this figure. $L+$ and $R+$ indicate left and right positions of the $S+$; $p$ gives the proportion of positive color in the $S+$. Right side: Linear regression functions of discrimination performance versus proportion disparity ratio with the two types of arrays.

behavioral performance and proportion values, stimulus values were expressed on a ratio scale. Disparity ratios have been used as a measure of relative numerosity (Boysen, Berntson, Hannan, \& Cacioppo, 1996) and were considered an appropriate measure of relative quantities here since discrimination of color proportion was expected to conform to Weber's law. The results of Experiment 1 could not distinguish between discrimination of relative versus simple differences in color proportion, so Experiment 2 tested whether use of a disparity ratio scale was justified.

The stimuli were horizontal bars of continuous blocks of color. Those on reinforced trials were the familiar ones with complementary color proportions. In nonreinforced test trials, the proportions of positive color were no longer complementary within a stimulus pair. Two sets of test stimuli were created. In one set, one bar had a proportion value corresponding to an S+ stimulus in Experiment 1 . The other bar always had equal proportions of positive to negative color. If the pigeons generalized from their previous training to choose the stimulus with the greater proportion of positive color, they should choose the bar with the $\mathrm{S}+$ proportion value. In the other set, a bar with equal color proportions was combined with one with a proportion value corresponding to an $S-$ stimulus in Experiment 1. On these trials, the pigeons should choose the bar with the equal color proportions, since it would contain the greater proportion of positive color. Note that, in Experiment 1, stimuli with the .5 value had always been paired and so had been indiscriminable. Since the pigeons had performed at chance on those trials, the .5 stimulus value had been equally often associated with reward and time-out. By itself, it should elicit neither strong approach nor avoidance responses.

If the pigeons discriminated color proportions as disparity ratios, the data points from the three stimulus sets should all fall along a straight line when logit performance is plotted as a function of proportion disparity ratio. The performance levels with the two test sets should differ, however, since the disparity ratio values for test stimuli diverge between sets. If, instead, the pigeons discriminate simple proportion differences, their performance should be the same across the test sets, which contain identical values for absolute proportion differences.

\section{Method}

\section{Subjects and Apparatus}

The subjects and apparatus were the same as in Experiment 1.

\section{Procedure}

There were eight sessions, combined into four pairs with counterbalanced stimulus parameters. Each session consisted of 102 trials. The first 6 were warm-up trials with uniform red and green horizontal bars (each $50 \times 10$ pixels, with a $36 \times 39 \mathrm{~mm}$ target area). In this and subsequent experiments, the warm-up trials were the same as those used at the start of Experiment 1, so data from these trials will be ignored. For the remaining 96 trials, half were reinforced training trials, and the other half were nonreinforced test trials. The stimulus pairs used on these trials are illustrated in Figure 4.

On the training trials, the stimulus parameters were the same as those used with solid horizontal bars in Experiment 1. For each proportion value, there were 8 training trials per session. Two sets of 
Training Stimuli S+/S-

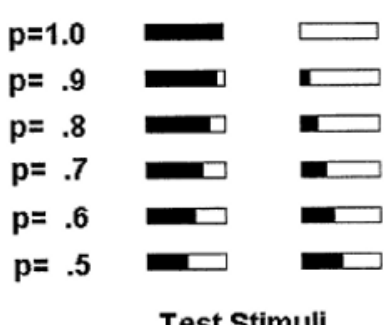

Test Stimuli

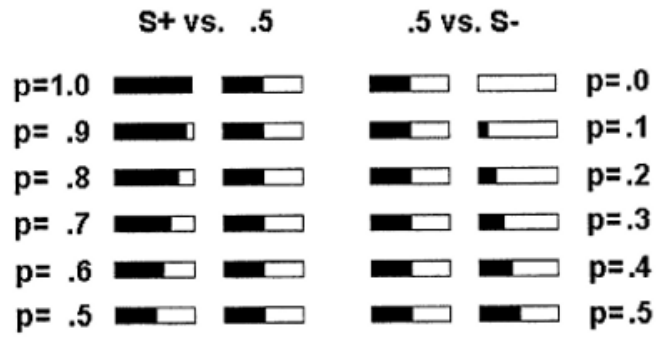

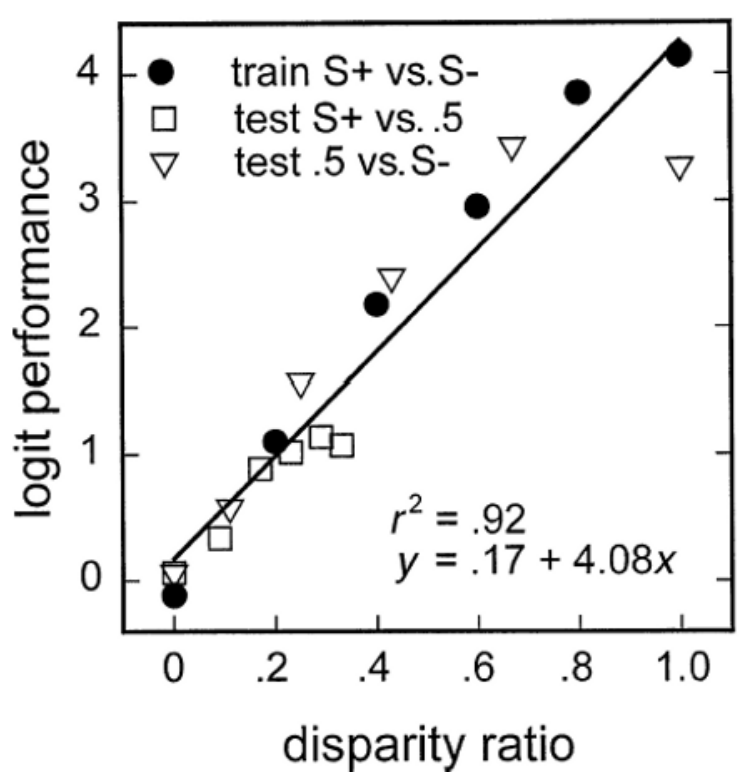

Figure 4. Left side: Examples of stimuli used for training and test trials in Experiment 2. As in Experiment 1, there were four configurations for the positions of positive- and negative-color portions in mixed-color stimuli. Only the L/L configuration is shown here. Left $p$ values indicate the proportions of positive color in the $S+$ stimulus for the training set and in the "correct" stimulus for the $S+$ versus .5 test set. Right $p$ values show the proportions of positive color in the "incorrect" stimulus for the .5 versus $S-$ test set. For illustration purposes, the correct stimulus is shown on the left in each pair. Right side: Linear regression function from mean discrimination scores for all training and test trials.

stimuli were used on test trials. For one set, the proportion of positive color in one bar corresponded to one of the six $\mathrm{S}+$ values $(1.0$ to .5), whereas the proportion value in the other bar was fixed at .5 . Choice of the $\mathrm{S}+$ value was defined as correct. For the other set, one bar had a positive-color proportion with one of the values of the $\mathrm{S}-$ stimuli in training trials ( 0 to .5 ). Choice of the other bar with a .5 proportion value was scored as correct. Both sets of test stimuli contained pairs in which one bar with the .5 value was arbitrarily coded as correct. There were four configurations of the red and green stimulus portions. To fully counterbalance all the parameter combinations (proportion $\times$ bar configuration $\times$ correct location) for the test trials, 48 trials were required for each set of test stimuli. The full complement of trials was equally distributed across two consecutive sessions. Per session, there were 4 trials for each of the six combinations of proportion values in each test set. This gave 8 trials with stimuli having identical .5 proportion values. The choices on these trials were pooled across the 16 trials in paired sessions. For each set of test stimuli, the same score was used for this proportion value in order to balance the design in the data analysis.

On nonreinforced test trials, choice of either stimulus terminated the trial. The stimuli were extinguished, and the ITI began immediately. The order of training and test trials was quasi-random, with equal numbers of training and test trials in each block of 12 trials and no more than 4 nonreinforced test trials occurring consecutively. The order of trials with the varying stimulus parameters was also quasi-random. Four different coding sequences controlled trial order and were changed daily for each pigeon.

\section{Results}

Scores for each stimulus set (training, $\mathrm{S}+/ .5, .5 / \mathrm{S}-$ ) were combined across the paired sessions. Scores were initially pooled across proportion values within each stimulus set, since these values were not the same across sets.
Stimulus configuration was disregarded in this analysis, since it did not affect discrimination accuracy in Experiment 1. A two-way ANOVA was applied to the pooled scores with stimulus sets (training, $\mathrm{S}+/ .5, .5 / \mathrm{S}-$ ) and sessions (four pairs) as the main factors. The only significant difference was in mean discrimination scores across stimulus sets $[F(2,10)=40.57]$. Newman-Keuls post hoc comparisons showed that performance with the $\mathrm{S}+/ .5$ stimulus set $(M=67.4 \%)$ was significantly worse than that with both the training and the $.5 / \mathrm{S}-$ sets $(M \mathrm{~s}=$ $84.6 \%$ and $80.7 \%$, respectively). There was no significant difference between the latter two stimulus sets.

Logit values were computed from the mean percent correct scores at each of the six proportion combinations per stimulus set, and disparity ratios were calculated for each proportion combination. The relationship between the logit measure of performance and the disparity ratios of positive-color proportions was tested with a linear regression analysis applied to the data from all three stimulus sets. The coefficient of determination was highly significant $\left(r^{2}=.92\right.$; see Figure 4$)$. Regression formulas, calculated separately for the three stimulus sets, were all significant and gave similar slopes and intercepts (slopes were $4.3,3.4$, and 3.5 , and intercept values were $0.2,0.1$ and 0.5 for the training, $\mathrm{S}+/ .5$, and $.5 / \mathrm{S}-$ sets, respectively).

\section{Discussion}

The main finding was of differences in discrimination scores with the three sets of stimuli. Mean performance 


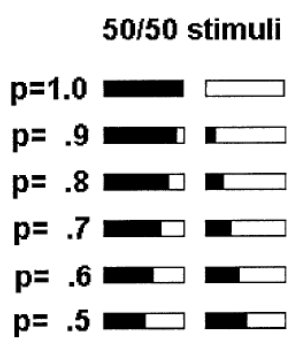

$50 / 100$ stimuli

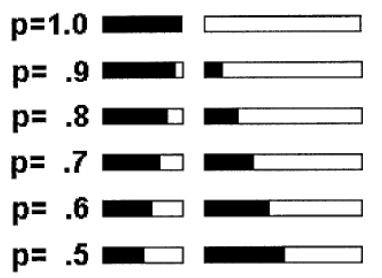

$100 / 50$ stimuli

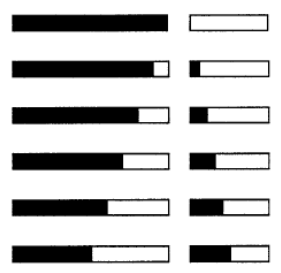

Figure 5. Stimulus sets, denoted by bar lengths in pixels, shown in Experiment 3. 50/50 stimuli were used on reinforced training trials. The other stimuli occurred on test trials. Proportions were complementary in paired bars, so $p$ values are for the proportion of positive color in the $S+$ or "correct" test stimulus. The correct stimulus is shown on the left. Only one configuration $(L / L)$ for the positions of positive and negative colors is illustrated.

was similar with training and $.5 / \mathrm{S}-$ test stimuli but was poorer with the $\mathrm{S}+/ .5$ test set. These differences tally with the mean proportion disparity ratios among the stimulus sets that were .5 for training stimuli, .18 for $S+/ .5$ test stimuli, and .41 for $.5 / \mathrm{S}$ - test stimuli. (If, instead, absolute differences in the proportions of positive color were used, the mean proportion differences for both sets of test stimuli would be .25; so average discrimination scores should have been similar.)

The significant result of the linear regression analysis supported the hypothesis that the pigeons discriminated the ratio difference in the proportion of color in each stimulus pair. However, since the size of the stimulus bars was constant, the pigeons could still have based their choices on relative differences in the lengths, or areas, of the positive-colored portions of the bars. Experiment 3 tested whether color proportions were still discriminated when the lengths of horizontal bars varied.

\section{EXPERIMENT 3}

In Experiment 3, the stimuli were horizontal bars, and the proportion values varied in the same way for both training and test stimuli. However, on test trials, the lengths of some of the bars were novel. Test trials were of three types. In one set, stimulus lengths were equal, but both bars were twice as long as the familiar training bars. In another set, the bar with the greater proportion of positive color in it was twice as long as the bar with the smaller complementary proportion of positive color. The latter bar was the same length as a training bar. In the third test set, the reverse was true. If the pigeons discriminated relative proportion independently of any other stimulus parameters, particularly of length or area of the positive-color component in paired bars, then their performance should be unaffected by these changes.

\section{Method}

\section{Subjects and Apparatus}

The subjects and apparatus were the same as in Experiments 1 and 2.

\section{Procedure}

There were 102 trials per session. The first 6 were warm-up trials with uniformly colored bars. In the remaining 96 trials, the proportion values for the correct and incorrect stimuli were the same as in Experiment 1. All the bars in this experiment were 10 pixels wide. On half the trials, the bars were 50 pixels long. The stimulus lengths $50 / 50$ were used to denote these training trials. Correct choices were reinforced with food reward, and incorrect choices led to time-out. For stimuli with identical proportion values, one was arbitrarily coded as correct. The other 48 nonreinforced test trials were intermixed with the training trials. On these trials, a peck to the bar with the greater proportion of positive color in it counted as correct. On 16 of the test trials (type 100/100), both bars of a pair were 100 pixels long. At .5 proportion values, one stimulus was again arbitrarily coded as correct. On another 16 trials (type 100/50), the correct bar was 100 pixels long, and the incorrect bar was 50 pixels long. At proportion values of .5, the 100-pixel bar was coded as the correct one. On the remaining 16 test trials (type 50/100), the correct bar was 50 pixels long, and the incorrect bar was 100 pixels long. For .5 proportion values, the 50-pixel bar was coded as the correct one. The touch-target area was $72 \times 39 \mathrm{~mm}$ for all stimuli. Stimulus sets are shown in Figure 5.

Counterbalancing of all stimulus parameters ( 6 proportion values $\times 4$ color configurations $\times 2$ side locations for the correct stimulus) in each of the three test sets was achieved across triplets of sessions. There were 12 sessions in all. The $\mathrm{S}+$ training stimuli and the correct test stimuli appeared equally often on the left and right sides within each session. There were six different sequences, changed daily, for controlling trial order.

\section{Results}

Scores were pooled across session triplets to calculate the percent correct choices for each proportion value within each stimulus set (50/50 training set and 100/100, $100 / 50$, and 50/100 test sets). These data were examined with a three-way (stimulus sets $\times$ proportion values $\times$ session triplets) ANOVA. All main factors had significant effects on performance. Reliable differences in discrimination accuracy were found among the four stimulus sets $[F(3,15)=6.60]$ and across proportion values $[F(5,25)=69.74]$. However, mean performance level also changed across session triplets $[F(3,15)=8.05]$. Furthermore, some interactions were significant. These included stimulus set $\times$ proportion $[F(15,75)=3.75]$ and stimulus set $\times$ session triplets $[F(9,45)=12.29]$. A Newman-Keuls post hoc test showed that, for the stimulus sets, scores on training trials (mean \% correct $=87.1$ ) were significantly better than scores with each test set. The scores with the three test sets did not differ reliably among themselves (mean $\%$ correct $=71.2,73.5$, and 
78.2 for $100 / 100,100 / 50$, and 50/100 sets, respectively). When the same post hoc test was applied to the factor of session triplets, the first triplet (mean \% correct $=84.0$ ) scored significantly better than each of the remaining three. On average, performance across these last three triplets was not significantly different $(78.6 \%, 73.5 \%$, and $74.0 \%$ for the second, third, and fourth triplets). Finally, the three-way interaction of proportion $X$ stimulus set $X$ sessions was also significant $[F(45,225)=2.17]$. This complex interaction prompted a closer examination of the test results.

Data from familiar reinforced training trials $(50 / 50$ set) were examined with a linear regression of logit discrimination scores versus disparity ratios of color proportions. The coefficient of determination for these training trials was significant $\left(r^{2}=.86\right)$. The smaller $r^{2}$ value, compared with that in the previous experiments, was due to high discrimination accuracy, approaching ceiling, at intermediate and high disparity values. Nevertheless, performance on training trials was fairly consistent across test sessions (see Figure 6). By contrast, scores on nonreinforced test trials changed across sessions.

Experiment 2 showed that the pigeons' performance was related to the disparity ratio of color proportions (leaving open the possibility that the pigeons judged area or length differences). So a disparity ratio scale was used again to assess the data in Experiment 3. However, plots (not shown here) of the pigeons' choices against either the disparity ratio of positive-color proportions or the disparity ratio of the lengths of these positive-color parts produced different types of discrepancies. These were difficult to reconcile with a simple discrimination of either color proportion by itself or color length (or area) alone.

The disparity ratio of color proportions was defined, as before, as the difference in proportions of positive color in $\mathrm{S}+$ and $\mathrm{S}-$ divided by the sums of those proportions. The range of proportion disparities (between 0 and 1) was the same for each stimulus set. If the scores were plotted against this disparity ratio scale, performance level in all sessions generally declined with a decrease in proportion disparity for each stimulus set. However, even in the first session triplet, the plots for the four stimulus sets did not match exactly. Although the results for the $50 / 50$ training stimuli and the 100/50 test stimuli were similar, choice performance was poorer at intermediate proportion disparities with 100/100 stimuli. Some of the scores with the 50/100 stimuli were also deviant. The lowest scores for this test set $(45.8 \%$ and $35.4 \%$ correct choices) were lower than expected (and than the other empirical test results) for proportion disparities with a small, positive value (.2) or a zero value.

To see whether the pigeons had instead discriminated relative differences in the lengths (or areas) of the positivecolor parts of paired bars, the data were plotted against a scale of the disparity ratios of positive-color lengths. This form of disparity ratio was calculated as the difference in pixel lengths of the positive-color portions in S+ and Sdivided by the sums of those lengths. Disparity ratios calculated in this way ranged from 0 to 1 for same-length stimuli (50/50 and 100/100 sets). However, for the 50/100 set, the range of ratio values was -.33 to 1 , and, for the $100 / 50$ set, it was .33 to 1 . With this method of plotting, there was also a general decrease in correct choices with a decrease in length disparity, but other discrepancies appeared. In particular, in the initial sessions, the lower scores $(45.8 \%$ and $35.4 \%)$ in the $50 / 100$ test set were now higher than expected for their respective disparity ratios based on the positive-color lengths $(-.14$ and -.33 ). Another deviant data point was produced in the $100 / 50$ test set for which scores at the smallest length disparity ratio (.33) dropped to a much lower level than those for bar pairs with similar disparity ratios in the other stimulus sets. (This, by the way, was not the case if disparity ratios for this set were based on color proportions.)

A better fit to the initial interaction data was obtained by assuming that the pigeons' choices were based on relative differences in both the proportions and the lengths of color portions. As a first approximation of this idea that the pigeons used both cues, values were calculated of the averaged disparity ratios of positive-color proportions and of the lengths of positive-color parts in stimulus pairs. For example, for stimuli in the $100 / 50$ set with proportion values .7 versus .3 , the disparity ratio of positivecolor proportions was .4 , whereas the disparity ratio of positive-color lengths was .65. In this case, the averaged disparity ratio was .525 . The pigeons' choices across the test session triplets are plotted against this averaged disparity ratio scale in Figure 6. This figure illustrates how the results on test trials changed across session triplets. An additional assumption to explain these changes is discussed below.

\section{Discussion}

Although the data from training trials yielded a slightly lower coefficient of determination than in the previous experiments, this was due to the increased discrimination accuracy at some of the intermediate proportion values. This enhanced performance is perhaps not surprising since, by this stage, the pigeons were highly familiar with these training stimuli. Otherwise, the pigeons' discrimination accuracy on reinforced trials remained fairly stable across the test sessions of this experiment. This was not the case with their choices on nonreinforced test trials, as the various interaction effects showed. Overall, a change in length in one or both stimuli reduced the pigeons' performance level, so their choices could not have been based on differences in color proportions alone. This applied even in the initial triplet of test sessions, where the data were most likely to reveal which stimulus factors guided the pigeons' spontaneous choices. On the other hand, the pigeons did not seem to have based their choices on a discrimination of positive-color lengths (or areas) alone.

Another possibility was that the pigeons utilized both disparities in color proportions and in lengths of the positive color. This was the reason for averaging the disparity 


\title{
session triplets:
}
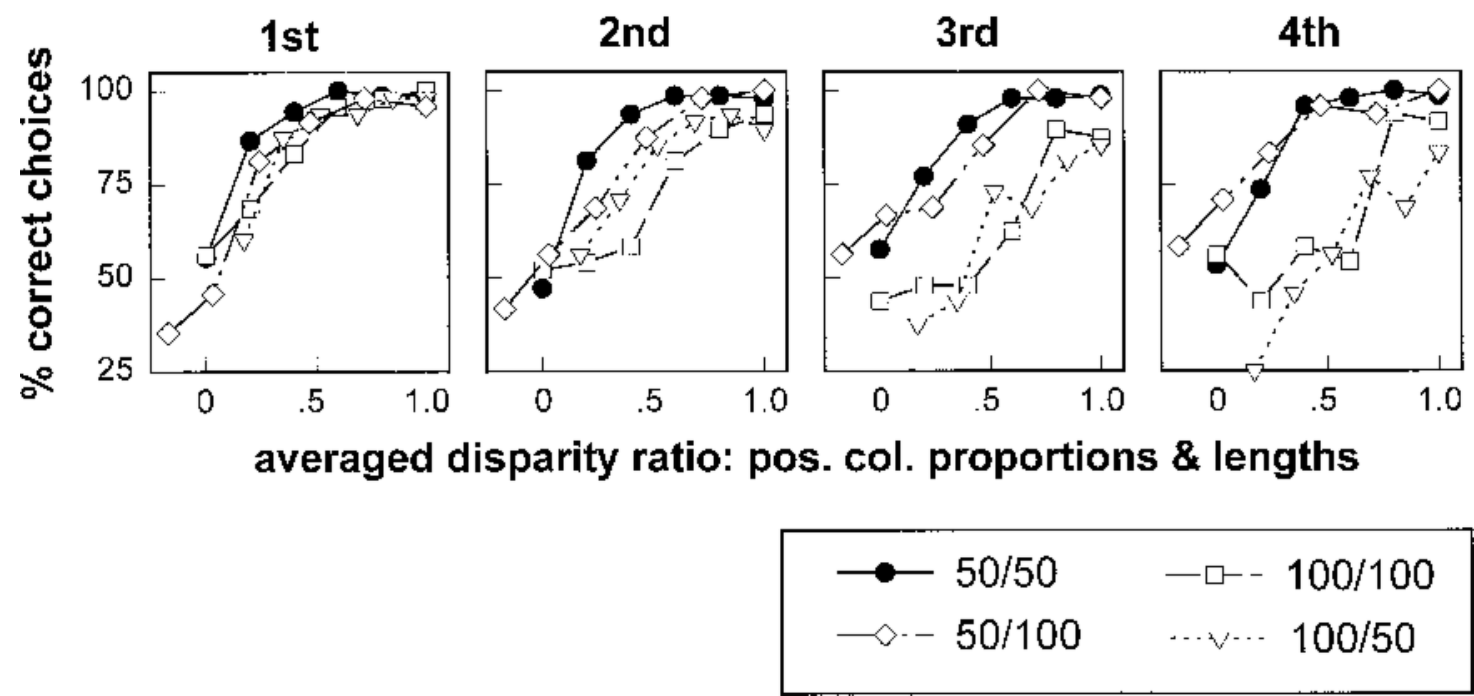

\begin{abstract}
Figure 6. Results across session triplets for the training and test trials in Experiment 3. Choice scores are shown on a percent correct rather than logit scale, since several of the scores reached $100 \%(=\operatorname{logit} \infty)$. For explanation of the averaged disparity ratio scale, see text.
\end{abstract}

ratios based on color proportions and on positive-color lengths. Plotting the data from the various stimulus sets against this averaged disparity scale produced a better match among the scores from the initial test sessions (see left-hand graph of Figure 6). The pigeons might have relied on both factors each time they made a choice or they may have wavered between these two factors across trials.

Although assuming that the pigeons assessed disparities in color proportion and in positive-color lengths resolved some of the problems in interpreting the initial test results, it still did not explain the changes across sessions. On 100/100 trials where neither bar length was familiar, choices became more erratic, and performance level progressively dropped below that on most of the $50 / 50$ training trials. The largest decreases in performance were on trials with 100/50 stimuli. On the other hand, performance at the lower disparity values on 50/100 trials improved across sessions. In part, these results could have been due to the pigeons' being biased to choose the familiar 50-pixel bar length, whereas they increasingly avoided the 100-pixel test stimuli. However, replacing the S+ by a 100-pixel bar led to a steady decline in performance on test trials (100/50 and 100/100), whereas replacing the $\mathrm{S}-$ by a longer bar did not have this effect (compare 50/50 and 50/100 scores). This asymmetry in the test results suggests that the pigeons processed the $\mathrm{S}+$ and $\mathrm{S}-$ differently. What the test results also suggest is that, with extended experience with horizontal bar training stimuli, the pigeons had acquired information about multiple stimulus parameters. These parameters appear to include the relative proportions of red and green color, the relative lengths, or areas, of critical regions like the positive-color portions, and the overall size (length or area) of stimuli that were associated with reward.

\section{EXPERIMENT 4}

Experiment 4 examined more closely which components of the horizontal bar stimuli influenced the pigeons' choices. In nonreinforced test trials, they were confronted with novel stimulus pairs consisting of some of the red or green parts of the familiar $\mathrm{S}+$ or $\mathrm{S}-$ training stimuli. On some of these trials, the positive- or negative-color test bars were paired with a gray bar, a color that was novel to the pigeons. These stimuli provided another test of whether the pigeons responded according to the proportion values of red and green. If a bar had the positive color, its proportion value was 1 . If it had the negative color, its proportion value was 0 . If it was gray, it was assumed to be a neutral stimulus without a proportion value (i.e., it contained neither of the other color components and had acquired neither excitatory nor inhibitory properties).

The stimuli were also designed to test alternative hypotheses. Since the red and green test bars were actually components of training stimuli, their lengths varied. Several different length combinations were created to examine whether the pigeons chose according to either the absolute or the relative lengths of familiar positive and negative colors. Testing the pigeons' response to the absolute lengths of red and green stimuli would eliminate one remaining explanation of the initial choices with test stimuli in Experiment 3. Those choices could have been due to a discrimination of relative color proportion that was biased by the pigeons' choosing or avoiding specific 
pos. col. / neg. col.

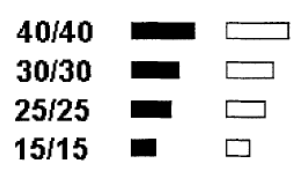

pos. / pos. col.

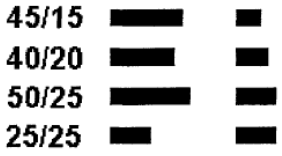

neg. / neg. col.

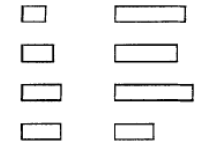

$15 / 45$

$20 / 40$

$25 / 50$

$25 / 25$

\begin{tabular}{|c|c|c|c|c|}
\hline \multicolumn{2}{|c|}{ pos. col. / gray } & \multicolumn{3}{|c|}{ gray / neg. col. } \\
\hline $50 / 50$ & $x \times x \times$ & $\square \times x \times$ & & $50 / 50$ \\
\hline $35 / 50$ & $\infty x$ & 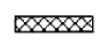 & $\square$ & $50 / 35$ \\
\hline $25 / 50$ & 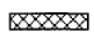 & $x \times \infty$ & $\square$ & $50 / 25$ \\
\hline $15 / 50$ & $\infty x \times x$ & $\$ x \times x>0$ & $\square$ & $50 / 15$ \\
\hline
\end{tabular}

Figure 7. Test stimuli used in Experiment 4. Black bars represent stimuli in the positive color, white bars represent those in the negative color, and cross-hatched bars represent those in a gray color. The numbers beside each stimulus pair give the bar lengths in pixels. For illustration purposes, the "correct" stimulus in each test pair is shown as the left one.

color components normally found in familiar training stimuli. This seemed an unlikely possibility. It was one that the results of Experiment 4 could eliminate.

Finally, Experiment 4 also investigated whether the pigeons gave equal weight to positive- and negative-color components of training stimuli.

\section{Method}

\section{Subjects and Apparatus}

Only 5 of the pigeons used previously served as subjects, since 1 had died after Experiment 3 was completed. The apparatus was the same as before.

\section{Procedure}

Six sessions were completed, each consisting of 96 trials. The first 8 were warm-up trials with one red and one green horizontal bar. Target areas were $30 \times 39 \mathrm{~mm}$. There were 48 reinforced training trials with stimuli that were $50 \times 10$ pixels in size and in the same color proportion values as in Experiment 1 . The other 40 trials were nonreinforced test trials with five sets of test stimuli (Figure 7). Bar width was always 10 pixels, but bar length varied. Within each set, there were four different pairs of test stimuli. Each pair appeared on 2 trials per session to counterbalance the left/right stimulus positions. Training and test trials were presented in quasirandom order. Two different sequences of trial order were used, and they were alternated daily.

In the first test set, one bar was in the positive and the other was in the negative color, so the relative proportion of one color to the other was always 1:0. If the pigeons relied only on proportion, they should always choose the positive-colored stimulus with the same high level of accuracy. Choices of the positive-color bars were scored as correct. However, the bar lengths on different trials were $40 / 40,30 / 30,25 / 25$, or $15 / 15$ pixels per stimulus pair. The positivecolor bars corresponded to parts of $\mathrm{S}+$ training stimuli with proportion values of $.8, .6$, and .5 and an $S-$ value of .3 , respectively.
The negative-color bars were components of the corresponding $\mathrm{S}-$ or $\mathrm{S}+$ training bars. If the pigeons had learned the absolute lengths of individual components, their choices should have declined from above chance for the 40/40 test pair to below chance for the $15 / 15$ pair.

In the second test set, both bars had the positive color, but their lengths varied in the combinations 45/15, 40/20, 50/25, and 25/25. Any length greater than 25 was a component of an S+ training stimulus, and any length less than 25 was a component of an $\mathrm{S}-$ training stimulus; the length 25 was part of the .5 proportion $\mathrm{S}+$ and $\mathrm{S}-$ stimuli. If the pigeons discriminated solely on the basis of color proportions, they would have to choose between test bars in which the proportion values were both 1 , so their choices should be randomly distributed. If they had learned about the specific lengths of the separate color components, their choices on the first three length combinations should be directed toward the longer bar and randomly distributed on the $25 / 25$ combination. The latter choice distribution would also occur if they assessed length disparities of the positive color. Choices of the longer bars were scored as correct, with arbitrary coding for the $25 / 25$ combination.

In the third set, both bars had the negative color, and length combinations were $15 / 45,20 / 40,25 / 40$, and $25 / 25$. Any length less than 25 was a negative-color component of an $\mathrm{S}+$ training stimulus, and any length greater than 25 was part of an $\mathrm{S}-$ training stimulus; the length 25 was again a component of indiscriminable training stimuli. So, if the pigeons' discrimination was based on proportion alone (here a value of 0 for both stimuli in a pair), they should choose randomly. If the lengths of components were important, and the pigeons had acquired information about both positive- and negativecolor components, their choices should be the converse of those on the previous test set. Choices of the shorter bars were scored as correct, with arbitrary coding for $25 / 25$ trials.

For the fourth set, one bar was always a gray one, 50 pixels long, whereas the other bar was positive colored, but its length varied. Length combinations were 50/50,35/50, 25/50, and 15/50. If the pigeons had learned only about color proportions, they should always choose the positive-color bar, and those choices were scored as correct. If they had learned about the absolute lengths of color components, they should strongly prefer the positive-color bar in the $50 / 50$ and $35 / 50$ pairs, since the positive-color bars were parts of S+ stimuli. They should weakly prefer it on $25 / 50$ trials, since that length of positive color occurred equally often on S+ and Straining trials and so was associated with partial reward, whereas the gray bar had no reinforcement history. On 15/50 trials, they should prefer the gray bar, since that positive-color length occurred in $\mathrm{S}-$ training trials.

For the fifth test set, the 50-pixel gray bar was paired with a negative-color bar of variable length, giving combinations of 50/50, $50 / 35,50 / 25$, and 50/15. If color proportion determines choices, the pigeons should always avoid the negative-color bar (with a proportion value of 0 ), so choices of the gray bars were scored as correct. If the pigeons had learned about the lengths of negative-color components in training stimuli, then the predictions about choice allocations are almost the opposite to those made for the fourth test set. They should strongly avoid the negative-color bar for length combinations 50/50 and 50/35. On 50/25 trials, the negative component, like the positive one, was associated with partial reinforcement on training trials and so should be weakly preferred to the gray bar. There should be a stronger preference for the negative-color bar on $50 / 15$ trials, since this length of negative color is normally a constituent of S+ training trials with a proportion value of .7.

\section{Results}

A two-way (proportion values $\times$ sessions) ANOVA was applied to the discrimination scores from training trials. Proportion changes significantly affected perfor- 


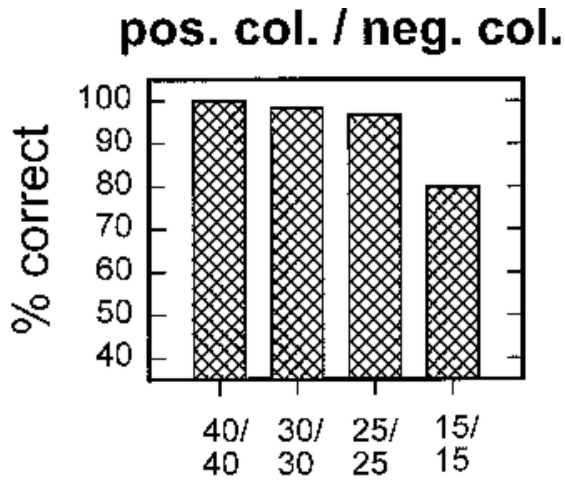

pos. col. / pos. pos. neg. col. / neg. col.
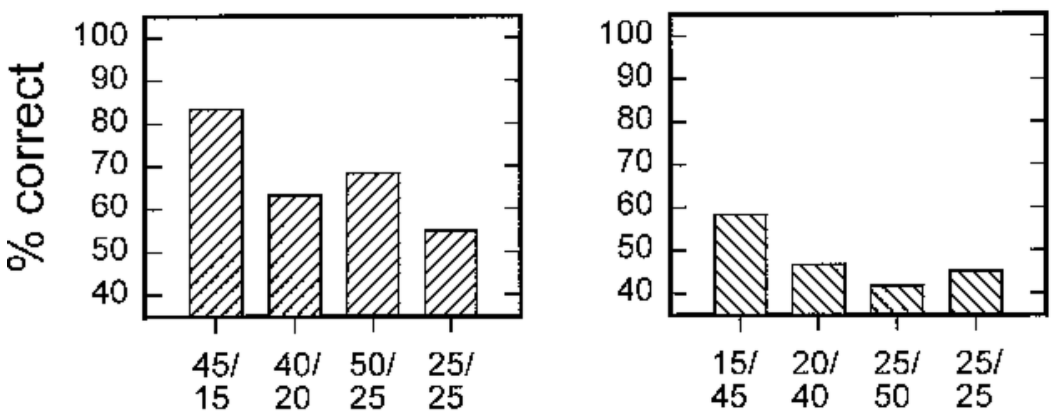

pos. col. / gray
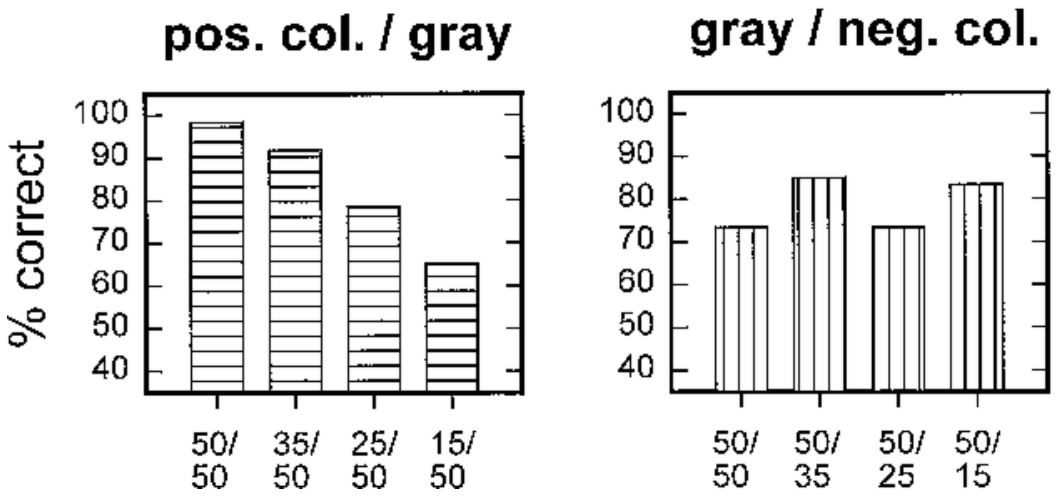

Figure 8. Mean discrimination scores (percent choice of "correct" stimulus) with the different sets of test stimuli in Experiment 4. Numbers beneath each histogram bar show the lengths, in pixels, of the "correct" and "incorrect" test stimuli for the various trial types within each test set.

mance accuracy $[F(5,20)=48.58]$, but sessions did not. A linear regression analysis of mean discrimination scores on a logit scale versus proportion disparity ratios yielded a significant coefficient of determination $\left(r^{2}=.91\right)$.

The data from test trials were also analyzed. Since the various trial types were not equivalent across test sets, the scores were first collapsed across trial types within each set. A two-way (test set $\times$ sessions) ANOVA revealed significant differences among the five test sets $[F(4,16)=$ 9.70]. Overall, there were no reliable changes across test sessions, but there was a significant interaction between test sets and sessions $[F(20,80)=2.33]$. These effects were examined in more detail with two-way (trial type $\times$ sessions) ANOVAs on the data from separate test sets. The mean data for the four trial types within each of the five sets are shown in Figure 8.

For the first test set, the means showed the pigeons' clear preference for choosing the positive-color stimulus over the negative-color one at all bar lengths. Although the percent correct score (choice of the positive-color bar) was lower for the trials with the shortest bar lengths $(15 / 15)$, an ANOVA showed no reliable differences in scores across the four trial types $(F<2)$. Choices did not consistently change across sessions $(F<1)$. 
In the second set, the greater the difference in relative bar length of positive-color stimuli, the more likely it was that the pigeons chose the longer bar, whereas, with equal bar lengths, choices were more or less random. These differences in scores across trial types were significant $[F(3,12)=3.72]$. There were also significant changes in choice allocations across sessions $[F(5,20)=5.03]$ but no significant interaction of trial types and sessions $(F<2)$. In the first two test sessions, the pigeons chose randomly on average (55\% and $50 \%$ correct choices); however, as testing proceeded, the mean preference for the longer bars progressively increased (to about 78\%).

In the third set, the mean choices between two negativecolor bars fluctuated around chance level, irrespective of relative bar lengths. The ANOVA showed no reliable differences among trial types $(F<2)$. Scores also did not change consistently across sessions $(F<2)$.

On the fourth test, the majority of choices were directed toward the positive-color bar rather than the gray one. The longer the positive-color bar, the more likely it was to be chosen. However, differences in test scores related to this bar's length were only of borderline significance $[F(3,12)=3.41, p=.05]$. There were no consistent changes in mean scores across test sessions $(F<1)$, but there was a significant interaction between trial types and sessions $[F(15,60)=1.92]$. At the longest lengths, the positive-color bars (trial types 50/50 and 35/50) were clearly preferred (between $80 \%$ and $100 \%$ ) to the gray ones across all sessions. On 25/50 trials, the positivecolor bar was always preferred, but choice level fluctuated across sessions. Choices of the shortest positive-color bar (trial type 15/50) tended to decrease, with some variability, from $80 \%$ to $60 \%$ across repeated sessions.

In the fifth test set, the pigeons showed an above-chance preference for the gray bar rather than the negative-color one. The differences in mean scores across different trial types were not significant $(F<2)$, and there were no reliable changes in test scores across sessions $(F<2)$.

\section{Discussion}

Discrimination accuracy on training trials was comparable to that in the previous experiments and was closely related to disparities in color proportions. The results of the various test sets suggest, on the whole, that the pigeons chose the positive color and avoided the negative color. The relative amount of positive color had more influence as test sessions progressed. However, there was no evidence that the pigeons had learned the absolute lengths of training stimulus components. In the first test set, the pigeons preferred the positive-color bar on all trials, including the 15/15 type. In the second test, the results over all the test sessions indicated that the greater the relative quantity of positive color, the more likely the pigeons were to choose the bar that was proportionally longer. But this effect emerged only over repeated sessions. At first, choices between the paired positive-color stimuli were distributed around a chance level. This initial result suggests that the pigeons first responded as if they were trying to choose between proportion values of 1 versus 1 but that they subsequently came to rely more on relative differences in positive-color length.

In the fourth test set, most of the choices were directed toward the positive-color bar rather than the novel gray one. The quantity of positive color in the different lengths of the positive-color bars had some effect on stimulus choices, but the differences in response patterns were only of borderline significance. Furthermore, these differences arose only over repeated sessions, since the preference for the positive color decreased just with small bar sizes.

The pigeons seemed to use the negative color as a generalized avoidance cue. Their choices in the third test between two negative-colored bars were random and were not influenced by the relative bar lengths. In the fifth test set, the pigeons generally avoided the negative-color bar, irrespective of its length, and instead chose the neutral gray bar, even though they had never been rewarded for choosing that stimulus. This failure to respond to changes in the quantity of negative color will be discussed below.

\section{GENERAL DISCUSSION}

This study demonstrated that pigeons can discriminate the relative quantities of colors in visual stimuli, even when these stimuli undergo substantial changes in their form. Although initially trained to perform this task with simple, horizontal bars of massed areas of color, the pigeons still accurately discriminated bars or arrays composed of randomly intermixed colored units. The array stimuli resembled those used by Honig and Stewart $(1989,1993)$, who equated changes in the proportions of colored items to changes in the relative numerosity of these elements. Because they employed a go/no-go procedure, and extinction tests of their pigeons' response to proportion changes, their results were more variable than the ones obtained here. But the overall trend in the data was similar in their experiments and in this one: Discrimination accuracy decreased as the proportions of two types of components approached .5 .

The question, though, is how the pigeons discriminated stimuli that were designed to vary in their color proportions. In the present training trials, stimulus size stayed constant and the color proportions were complementary in paired stimuli. In these training trials, and in several of the other stimulus conditions that were introduced, a nominal discrimination of proportion could have been achieved by comparing the lengths or summed areas of one or other color. Several of the experiments by Honig and Stewart (1989) suggested that their pigeons did not rely on area cues. They used arrays that were presented one at a time to pigeons in a go/no-go procedure. In one experiment the arrays consisted of red dots in two different sizes. Although their test data showed extinction effects, the pigeons' discrimination accuracy varied as a function of the proportion, or relative numerosity, of the two sizes of dots. This held true even when the total 
number of elements per array, and so the summed areas of larger versus smaller dots, was varied in test trials. Furthermore, the pigeons' discrimination of the proportion of mixed elements was unaffected when these elements consisted of different forms (e.g., stylized birds vs. flowers) for which integrated areas are unlikely to have provided a consistent basis for discrimination.

The results of Experiments 3 and 4 indicate that the pigeons could utilize relative differences in colored areas or, in bar stimuli, disparities in the lengths of one color component. However, they did not seem to rely solely on a comparison of such disparities. The test results of Experiment 3 were not explicable simply on the basis of disparities in lengths of the positive color. The analysis concentrated on the positive-color components, since examination of response distributions (not presented here) with the training stimuli in Experiment 1 had shown that the pigeons directed most of their pecks to the positivecolor portions of the bars. Plots of the initial choices made to test stimuli of varying lengths in Experiment 3 gave the best match when choice scores were plotted against a disparity scale that took into account differences both in proportions and in the lengths of positive colors in paired stimuli. This suggested that the pigeons could utilize information about both these factors. Furthermore, as testing under extinction continued, scores for different stimulus sets started to diverge. This was attributed to an increasing bias against a stimulus bar that differed in overall length from the familiar training stimuli. So the pigeons had apparently learned about stimulus size, too. Their flexibility in responding accurately when stimulus configurations were altered in Experiment 1 showed that it was not essential to maintain stimulus size or area constant, however.

Some of the test results in Experiment 4 also indicate that disparities between stimulus pairs in the lengths or areas of positive-color components played a contributory role but were not primary in determining the pigeons' choices. This was shown particularly when the pigeons had to choose between two positive-colorbars. Their initial choices were more or less random, and only as testing under extinction progressed did they show an increasing tendency to choose the longer of two bars. All these results suggest that the pigeons learned about multiple parameters of the stimuli and utilized several dimensions in judging which stimulus they should peck. This would actually be in line with other research on selective attention or visual categorization that also demonstrates multidimensional control (e.g., Chatlosh \& Wasserman, 1993; Jitsumori, 1996).

Because of the complementary amounts of color in all stimuli on reinforced trials, it might be expected that the pigeons gave equal weight to both color components, with the positive color acquiring excitatory properties and the negative acquiring equivalent amounts of inhibition. Experiments 3 and 4 revealed some asymmetry in the way the pigeons utilized the information available to them. Whereas the size of positive-color stimuli in the latter experiment had some influence on differential responding, the size of the negative-color stimuli did not, although the negative color had acquired general inhibitory properties. An asymmetry in what is learned about positive and negative features of discriminative stimuli has been reported when pigeons learn to categorize much more complex stimuli than the ones used here. Jitsumori (1996) constructed pictures of artificial butterflies to test for polymorphous categorization. Her pigeons learned more about the features characterizing the base pattern of the positive category than about those of the negative category. The apparent asymmetry in the use of stimulus cues in the present experiments concurs with selective attention theories of conditioning (e.g., Mackintosh, 1975). These theories assume that animals attend more to stimuli that are reliable predictors of reinforcement and that these stimuli acquire excitatory properties.

Even though the pigeons attended more to the parameters of the positive-color stimulus portions, this does not imply the pigeons were perceptually insensitive to the negative-color components. They must have assessed the extent of the negative-color portion(s) within a given stimulus in order to estimate the overall size of that stimulus. One way in which the pigeons might have computed the proportion of positive color, irrespective of absolute stimulus size or configuration, would be to assess the area occupied by the positive color within the area of the whole stimulus. If this operation is performed separately on each stimulus of a pair, the pigeon could then choose the stimulus with the greater proportion of positive color. Note that this proposal of area estimation within stimuli is different from the idea that the pigeons also discriminated the areas of just one color component between stimuli. Honig and Stewart's (1989) experiments indicated that stimulus area is irrelevant (unless their pigeons used the size rather than the color of stimulus components as cues for area summation). Under the training conditions in the present experiments, component areas were apparently more important, both for direct comparison and as a basis for assessing relative proportions of colors.

A further point that emerged from the present study, especially Experiment 2, was that the pigeons discriminated relative rather than absolute differences in stimulus values. The disparity ratio scale that was used is similar to one that has been applied to primates' numerosity discrimination (Boysen et al., 1996). The reliable linear relationship between logit performance and disparity ratio is what would be expected if Weber's law applies to the pigeons' discrimination of proportion differences. The way these birds differentiate this aspect of relative quantity resembles the manner in which pigeons and other animals assess numerical differences. Weber's law operates when animals are required to discriminate differences in the numerosity of either visual or auditory stimulus units or in the number of responses they themselves emit (Boysen et al., 1996; Fetterman, 1993; Gallistel \& Gelman, 1992; Meck \& Church, 1983). Animals' discrimination of temporal differences also obeys Weber's law (Gibbon, 1977; Meck \& Church, 1983). It seems likely that this law ap- 
plies to the way organisms differentiate a wide variety of stimulus attributes, ranging from the sensory to the cognitive. In this respect, Weber's law may well have the general applicability that the early psychophysicists supposed (Fechner, 1860/1966).

\section{REFERENCES}

Boysen, S. T., Berntson, G. G., Hannan, M. B., \& Cacioppo, J. T. (1996). Quantity-based interference and symbolic representations in chimpanzees (Pan troglodytes). Journal of Experimental Psychology: Animal Behavior Processes, 22, 76-86.

Brannon, E. M., \& Terrace, H. S. (2000). Representation of the numerosities 1-9 by rhesus macaques (Macaca mulatta). Journal of Experimental Psychology: Animal Behavior Processes, 26, 31-49.

BRown, P. L., \& JENKINS, H. M. (1968). Auto-shaping the pigeon's key peck. Journal of Experimental Analysis of Behavior, 11, 1-8.

Chatlosh, D. L., \& Wasserman, E. A. (1993). Multidimensional stimulus control in pigeons: Selective attention and other issues. In T. R. Zentall (Ed.), Animal cognition: A tribute to Donald A. Riley (pp. 271292). Hillsdale, NJ: Erlbaum.

Emmerton, J., Lohmann, A., \& Niemann, J. (1997). Pigeons' serial ordering of numerosity with visual arrays. Animal Learning \& Behavior, 25, 234-244.

FeCHNER, G. (1966). Elements of psychophysics (H. E. Adler, Trans.). New York: Holt, Rinehart \& Winston. (Original work published 1860)

Fellows, B. J. (1967). Chance stimulus sequences for discrimination tasks. Psychological Bulletin, 67, 87-92.

Fetterman, J. G. (1993). Numerosity discrimination: Both time and number matter. Journal of Experimental Psychology: Animal Behavior Processes, 19, 149-164.

FRISBY, J. P. (1979). Seeing: Illusion, brain and mind. Oxford: Oxford University Press.

Gallistel, C. R. (1989). Animal cognition: The representation of space, time and number. Annual Review of Psychology, 40, 155-189.
Gallistel, C. R., \& Gelman, R. (1992). Preverbal and verbal counting and computation. Cognition, 44, 43-74.

GibBON, J. (1977). Scalar expectancy theory and Weber's law in animal timing. Psychological Review, 84, 279-325.

Hollard, V. D., \& Delius, J. D. (1982). Rotational invariance in visual pattern recognition by pigeons and humans. Science, 218, 804-806.

HoNIG, W. K. (1992). Emergent properties of complex arrays. In W. K. Honig \& J. G. Fetterman (Eds.), Cognitive aspects of stimulus control (pp. 301-321). Hillsdale, NJ: Erlbaum.

Honig, W. K., \& STEWART, K. E. (1989). Discrimination of relative numerosity by pigeons. Animal Learning \& Behavior, 17, 134-146.

Honig, W. K., \& Stewart, K. E. (1993). Relative numerosity as a dimension of stimulus control: The peak shift. Animal Learning \& Behavior, 21, 346-354.

JITSUMORI, M. (1996). A prototype effect and categorization of artificial polymorphous stimuli in pigeons. Journal of Experimental Psychology: Animal Behavior Processes, 22, 405-419.

Mackintosh, N. J. (1975). A theory of attention: Variations in the associability of stimuli with reinforcement. Psychological Review, 82, 276-298.

Macmillan, N. A., \& Creelman, C. D. (1991). Detection theory: A user's guide. Cambridge: Cambridge University Press.

Meck, W. H., \& Church, R. M. (1983). A mode control model of counting and timing processes. Journal of Experimental Psychology: Animal Behavior Processes, 9, 320-334.

Pearce, J. M. (1997). Animal learning and cognition: An introduction (2nd ed.). Hove, U.K.: Psychology Press.

Roberts, W. A. (1998). Principles of animal cognition. Boston: McGraw-Hill.

Roberts, W. A., \& Mitchell, S. (1994). Can a pigeon simultaneously process temporal and numerical information? Journal of Experimental Psychology: Animal Behavior Processes, 20, 66-78.

(Manuscript received August 24, 1999; revision accepted for publication August 2, 2000.) 